\title{
An Exploratory Study of Input Configuration and Group Process in a Negotiation Task Using a Large Display
}

\author{
Jeremy P. Birnholtz ${ }^{1}$ Tovi Grossman ${ }^{2}$ \\ ${ }^{1}$ Knowledge Media Design Institute \\ University of Toronto \\ www.kmdi.utoronto.ca \\ $\{$ jeremy, clarissa.mak\}@kmdi.utoronto.ca
}

\author{
Clarissa Mak ${ }^{1}$ Ravin Balakrishnan ${ }^{2}$ \\ ${ }^{2}$ Department of Computer Science \\ University of Toronto \\ www.dgp.toronto.edu \\ \{tovi, ravin\}@dgp.toronto.edu
}

\begin{abstract}
This paper reports on an exploratory study of the effects of input configuration on group behavior and performance in a collaborative task performed by a collocated group using a large display. Twelve groups completed a mixed-motive negotiation task under two conditions: a single, shared mouse and one mouse per person. Results suggest that the multiple mouse condition allowed for more parallel work, but the quality of discussion was higher in the single mouse condition. Moreover, participants were more likely to act in their own best interest in the multiple mouse condition.
\end{abstract}

\section{Author Keywords}

Large displays, input, collaboration, negotiation

\section{ACM Classification Keywords}

H.5.2 [User Interfaces]: Interaction styles

\section{INTRODUCTION}

Large, wall-sized displays or combinations of displays have been used for many years for tasks such as meeting support [14], military control rooms [12], display-rich spaces [39], and social interaction $[15,16]$. More recently, advances in technology and falling costs of projectors and panel displays have enabled tiled displays (see Figure 1) that provide imagery at a much higher resolution than previously possible [35]. Such high-resolution large displays are not in widespread use but researchers have experimented with their use in, for example, collaboratively interpreting visualizations of scientific data [34, 48]. In addition to visualization, these displays arguably allow for novel forms of interaction involving more users and/or more information than traditional displays. Example tasks where such functionality might be useful include collaborative brainstorming, working with $2 \mathrm{D}$ and $3 \mathrm{D}$ design sketches where scale correspondence to the ultimate physical artifact is important, collaborative writing, or simply expanding the desktop [35] for a single user.

Permission to make digital or hard copies of all or part of this work for personal or classroom use is granted without fee provided that copies are not made or distributed for profit or commercial advantage and that copies bear this notice and the full citation on the first page. To copy otherwise, or republish, to post on servers or to redistribute to lists, requires prior specific permission and/or a fee.

CHI 2007, April 28-May 3, 2007, San Jose, California, USA

Copyright 2007 ACM 978-1-59593-593-9/07/0004...\$5.00.
Despite significant activity in the engineering of large highresolution displays, there is little consensus on how users should interact with them. Research systems have used mouse input [35], direct touch [5, 9, 36], freehand pointing [45], and laser pointers $[10,30,31]$, but none of these has emerged as a clear choice for a range of applications and indeed there may not be a single best option.

Moreover, when groups are using the display together, it is not clear how many sources of simultaneous input should be allowed. While single input allows only one person to work at a time, and thus may alter how a collaborative task is performed [19], its simplicity and backward compatibility to existing applications might be an advantage. In contrast, multiple inputs, while adding complexity at the system level, can allow for parallel work and may increase efficiency. While the "best" input configuration for a scenario likely depends heavily on the task and a range of social factors, it has also been shown repeatedly that users adapt their existing behavior to available technologies in ways that can influence process and outcomes [3, 32, 33]. Thus, it is also probable that input configuration might influence group behavior. Nonetheless, there has not been a systematic investigation into the effect of single vs. multiple input streams on group collaboration styles and outcomes when using a large, shared display.

In this paper, we present an exploratory study of groups performing a negotiation task on a shared high-resolution large display under two input configurations: single mouse and multiple mouse (one per user). We focus in particular on differences between these conditions in individual influence on negotiation, and group process and outcomes.

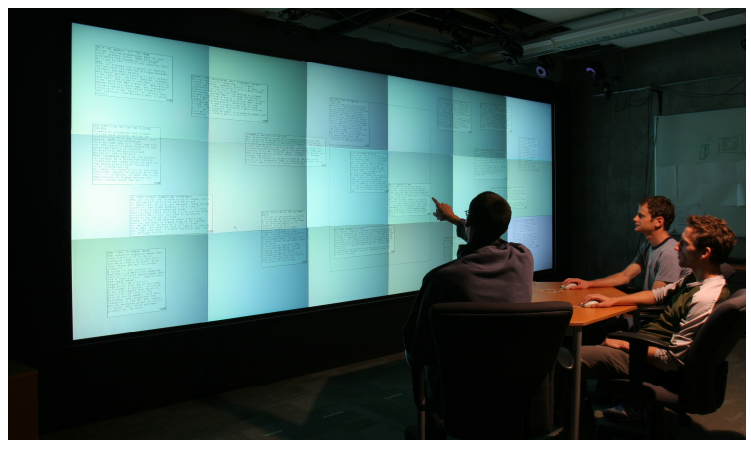

Figure 1. 5 x 1.8m high-resolution display used in this study. 


\section{BACKGROUND AND RELATED WORK}

\section{Large Display Input Modalities}

Researchers have explored various methods for interacting with large displays. However, there does not seem to be any consensus on which circumstances these different methods are appropriate for, or how these different modalities affect the users' experiences, especially in a collaborative setting.

A common method for interacting with a large display, particularly electronic whiteboards, is via direct stylus or touch input [14, 16, 27]. An obvious problem with such direct input is that the user may not be able to reach all areas of the display. Accordingly, various techniques have been proposed to reach the extent of the display when interacting directly on its surface[4, 6, 22, 30, 31].

Another common approach, and of greater interest to our current study, is interacting using traditional mouse input. This has the advantage that users can be comfortably seated, and at a distance that allows them to view the display in its entirety. Several techniques have been proposed to facilitate mouse input with large highresolution displays, such as improving the cursor visibility [4], warping the cursor between screen regions, and using a dynamic cursor size and control-display gain [2].

Less traditional methods, performed at a distance, include freehand pointing [45], using laser pointers [10, 30, 31] and multi-finger gestural input [24]. Such techniques may be useful for informal interactions with ambient displays [46] or for high degree-of-freedom tasks. However, these are likely to be less useful for more typical tasks such as creating or editing presentations or documents.

The abovementioned techniques were typically designed for a single user. It remains unclear as to what techniques are best suited for multiple users of large displays.

\section{Single Display Groupware}

There has, however, been some prior study of groups interacting with a shared smaller display using varying combinations and types of input modalities, largely in the area of Single-Display Groupware (SDG) [38, 42]. However, there has been little investigation into the impact of input modality and configuration on group process.

Inkpen et al. [19] studied pairs of children engaged in a play task, comparing a shared input device to a one-device-perperson condition. However, they provided only a single cursor in both conditions. Their results show that the more time a particular individual spent controlling the cursor (in both input conditions) the more skill that individual gained.

In work that also involved pairs of children, Stewart et al. [38] reported that children engaged in an educational task subjectively preferred two mice over a single mouse.

These studies provide some evidence to suggest that choice of input technique can impact group performance and satisfaction, but this question has not been investigated with adult participants engaged in non-play tasks.
DiMicco et al. [11], did study the impact of a large display on group conversation, but did not focus on input configuration or modalities. Vogt et al. [47] compared group performance with a mouse versus a laser pointer in completing a maze task, but did not examine influence on group behavior beyond speed of reaching a solution.

\section{Why might input matter?}

There are several reasons to believe that input configuration could impact group processes and outcomes in more significant ways, particularly with regard to individual influence on group negotiation and coordination processes.

\section{Opportunities for individual influence}

Social dominance is a trait studied in group psychology, particularly in tasks where negotiation is involved, such as the arctic survival task [13] or the one used in our experiment below. While certain people tend to be higher or lower on the social dominance scales than others, it has been shown that certain communication technologies can impact this. Huang [18], for example, showed that camera angle can influence perceived social dominance in a negotiation task performed using videoconferencing.

Burgoon [7] developed and validated a set of questionnaire scale items for measuring social dominance that consists of the following constructs:

Control of conversation: The extent to which any participant in a group or dyadic negotiation is perceived to monopolize the conversation and take charge.

Influence: The perceived degree of impact a participant has on others in winning them over to his or her point of view.

Panache: The perceived memorability and stylishness of a person's communication style.

Trust: The extent to which a person is perceived as reliable and truthful.

Poise: The extent to which a person is perceived as able to make decisions and take decisive action.

Self Assurance: The perception of a person's confidence.

When using a single shared mouse, it is possible that mouse possession will serve as a proxy for conversation control. In other words, a group member who is controlling the cursor will also be perceived as controlling the conversation. Mouse control in this scenario also gives the controller a potential persuasive edge over other participants in that the controller can carry out an action and then ask for approval or disapproval, whereas the others must first ask for the mouse or ask the controller to carry out an action for them.

However, it might also be that a single input device would make it more difficult for a group member to influence group outcomes. When multiple input devices are used, activities may take place in parallel. Given that human perception is typically focused on a single point of activity, it can be difficult to keep track of parallel streams of 
activity, particularly when these are spread across a large display space [1]. This stands in stark contrast to a single input device configuration in which focus is shared amongst group members, and activities carried out by one person are likely to be noticed by others. Thus, it may be harder for members involved in negotiation to act explicitly in their own best interest when there is a single input device.

\section{Group Process and Coordination}

Generally speaking, coordination involves the management of dependencies between activities [25]. In workgroups, this means managing available human, technology and capital resources to accomplish a shared goal. In HCI and $\mathrm{CSCW}$, much coordination research has focused on issues that arise in distributed groups or in groups using multiple input devices (e.g. [28]), such as coordinating pointing activities and handling simultaneous editing of documents.

There has been some work examining the effects of turntaking protocols on group outcomes with children using SDG systems [19], but little work examining the effect of input configuration on group process and coordination. It has been shown, however, that a range of factors related to the configuration of physical space can impact group interactions and behaviors with a tabletop display [20], so it stands to reason that input configuration should have some effect on group activities using large displays.

Van de Ven et al. [44] and Thompson [41] list multiple modes of work flow that play into coordination. Most relevant for our purposes are "independent" and "team" modes. In independent workflow, work is done largely in parallel by individuals, whereas in team workflow, the entire group works together to achieve a shared outcome. However, few groups work exclusively in one of these extremes. Uncertainties may arise in independent work that require clarification [44], or consensus may be reached in team interaction that then requires individual efforts to be completed in parallel. Thus, most groups move between states of independent and collective activity [23].

The ability of groups using SDG systems to move fluidly between these states, however, may be constrained by the technology. Groups provided with only a single input device cannot engage in independent, parallel work if the tasks involve any sort of interaction with the system. This means that tasks that would otherwise be completed in parallel must be done either sequentially (but individually), where the input device is passed from member to member, or a single member is asked by other participants to complete specific actions, or as a team, where the group does not divide up effort and discusses each action as it is carried out.

In either case, we would expect substantial effects of input configuration on group efficiency and process. The lack of ability to work in parallel should cause some slowdown, and the constrained mode of input should force discussion of group process.

\section{THE PRESENT STUDY}

In this study we seek to build on prior work in the areas described above by studying small groups (of 3 individuals) engaged in a "newspaper layout" negotiation task that involves visual elements on a large, high-resolution display and performed using two different input configurations. In one configuration, only a single mouse was provided, and it was up to the three participants to decide how to use it and share it amongst themselves. In the other condition, three mice were provided, one for each user, and they could be used to simultaneously control three independent cursors. We will examine how the groups use the display, and, in particular, how input configurations impact group process and perceived dominance within the groups.

\section{Participants}

Thirty-six people (9 female, 27 male), aged 18 to 30 participated in the experiment. Participants signed up for the experiment in groups of three, and were required to have known each other for at least 3 months, in order to avoid groups of complete strangers who might behave differently than those who already knew each other. All were required to have normal or corrected-to-normal vision, and to use English as their primary language. Participants were paid $\$ 10$, and had the opportunity to win an additional $\$ 5$ if they had the highest score in each of four trials. All groups successfully completed at least 3 trials.

\section{Setup and Equipment}

We used a 5 meters wide by 1.8 meters high single-surface, back-projected display. Eighteen 1024 x 768 pixel projectors arranged in a $6 \times 3$ matrix provide an effective resolution of $6144 \times 2304$ pixels. The display is driven by a cluster of $18 \mathrm{PCs}$, with distributed graphics rendering enabled by Chromium (chromium.sourceforge.net). Three standard two-button optical USB mice were used.

Because the task involves both reading the display and doing layout work with interaction, participants sat at a hexagonal table located 5 feet from the display (Figure 1). This configuration meant that all participants could see the display and each other with relative ease. The font size on the display was calibrated so that it was easily legible by a person with 20/20 vision at a distance of 8 feet.

\section{Task Description and Procedure}

Unlike prior studies that have used newspaper layout tasks [17], our intent was not to test layout tools or have participants work together in an entirely cooperative manner. Rather, our task was designed to more closely mimic real-world scenarios such as war rooms [40] and committee meetings where multiple stakeholders come together in a competitive environment to make decisions. The task involves elements that McGrath [26] would characterize as 'mixed motive negotiation' and 'decision making.' Participants were told that they had been assigned to be associate editors for different sections of a daily 
newspaper devoted to agricultural issues ${ }^{1}$. One participant was responsible for fruits, another for vegetables and the third for proteins. Each was then given a list of three keywords related to that topic (e.g., "banana," "coconut," and "grape" for the fruits editor). Each keyword had a specific point value assigned to it. Each editor's area of responsibility was known to the other two, but the keywords and their values were private.

Participants had the shared goal of laying out the front page of the next day's newspaper, selecting from a set of available articles, with the constraints that no more than 14 articles could be selected, articles could not overlap, and large areas of white space were not permitted. At the same time, each had the individual goal of maximizing his or her score. Points were earned each time one of a participant's keywords appeared in an article included in the final front page layout. For example, if the keyword "banana" was worth 3 points, the fruits editor would earn 9 points for an article in which the word banana appears 3 times.

Articles were based on real newspaper articles, but were modified to include the requisite keywords. Articles varied substantially in their value to each individual participant. Some were valuable only to one participant, while others were valuable to all three. Points were balanced across all articles such that no participant had a built-in advantage.

Participants practiced using the interface before the formal trials began. Then, groups had to complete a layout within 12 minutes, a limit found to be reasonable in our preliminary pilot studies. The procedure roughly involved the following steps:

- individual reading to determine articles of value

- selecting the most valuable articles

- group discussion of articles to go on the front page

- group discussion of how the page would be laid out

- group negotiation about what to keep/cut to stay within the task constraints.

\section{Design}

A within-subjects design was used in which each threesome performed the task twice using each of two input configurations, for a total of four trials per group. The order of conditions was balanced across groups. The experimental conditions were as follows:

Single mouse: The group was provided with a single mouse, placed in the center of the table before the experiment began. No explicit roles were assigned and it was up to the group to determine who would control the mouse and when.

Multiple mouse: The group was provided with three mice, with one placed in front of each user. Each mouse controlled a uniquely-colored cursor on the screen.

\footnotetext{
${ }^{1}$ Agriculture was chosen because our participants were unlikely to attach a priori importance to arbitrary agricultural issues in the way that they might if we had used, say, "international," "national," and "sports" news.
}

While participants were assigned responsibility for specific content, explicit roles for executing the task (e.g., mouse operator, process coordinator, etc.) were not assigned. Seating positions were randomly assigned and topic assignments to seating positions were fully counterbalanced throughout the experiment. Article set order was counterbalanced using a balanced latin square design.

\section{Layout Application}

The screen layout consisted of a rectangle centered on the display representing the front page borders, and rectangular articles which could be placed within this page. The articles were initially placed with a random position and size, with the constraints that no articles intersected, and articles were completely outside of the front page (see Figure 2).

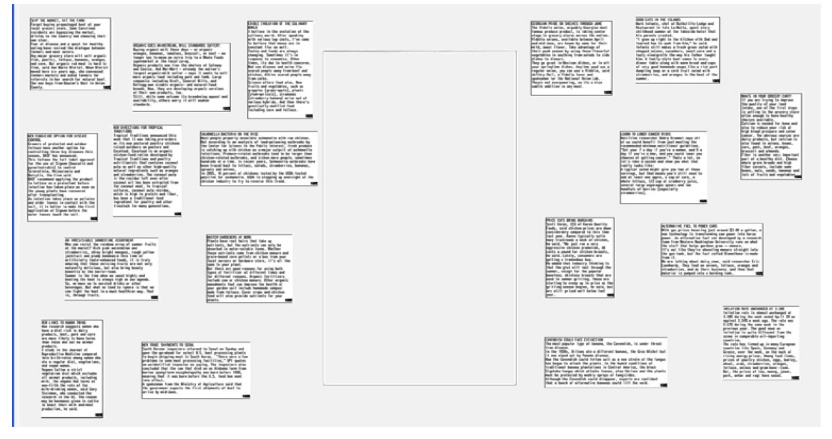

Figure 2. A screen-capture image of the layout application at the start of the experiment. Articles are scattered about the perimeter at random. The box in the center represents the front page of the newspaper.

Two standard interactions were supported - moving and resizing. To move an article, the user clicked within its borders at which point the position of the article was bound to the position of the cursor. The movement completed when the user released the mouse button. Resizing was accomplished by clicking on either an article's bordering edge, which allowed resizing by dragging that edge, or on an article's bordering corner, which allowed simultaneous resizing of the two corresponding adjacent edges by dragging. When both positioning and resizing, the article's edges snapped to an invisible grid, enabling easy alignment of articles with each other and to the front page borders.

Article content was always rendered to span the entire width of its containing rectangle. When articles were resized such that their containing rectangles were too small to display their entire content, the content which could not fit simply wasn't displayed. In the bottom right hand corner of each article the percentage of the currently visible content was displayed. When articles were made larger than required to display their entire content, extra void space was simply displayed after the end of the article.

In the multiple mouse condition, users controlled their own independent cursors, which were both color coded and numbered. Articles could only be manipulated by one cursor at any given time: as soon as one cursor clicked on an article, all other cursor input was ignored by that article. 


\section{Hypotheses}

With regard to the effect of input condition on social dominance, we hypothesized that:

- Individuals would be perceived as more dominant in the single-mouse than in the multi-mouse condition.

- Individuals would act in their best interest more in the single mouse than in the multi-mouse condition.

We also expected differences between the input conditions in terms of how the groups performed the task and their satisfaction with the outcome, and hypothesized that:

- Groups with a single mouse would have more discussion of group process.

- Groups with multiple mice would be more efficient in portions of the task involving independent work.

- There would be more parallel activity in the multi-mouse condition than in the single mouse condition.

- All members of groups would be happier with the outcomes in the multi-mouse condition.

\section{Event Recording and Analysis}

Analysis was carried out using data from event logs, questionnaires, and video recordings.

For each moving and resizing event we recorded the time at which it occurred, the user who carried out the event, the article which the event was performed on, whether the article was moved onto or moved off of the front page, the old and new dimensions and positions of the article, and the old and new scores of all three users. For the single mouse condition, the experimenter recorded each time the mouse was switched between users. This allowed us to determine how long each user controlled the mouse, and who was responsible for each of the other two types of events.

A video camera positioned to the right of the display, facing the three users, recorded all user activity throughout the experiment. A microphone was placed on the table to capture the conversations. A technical problem in recording meant that 5 of the video recordings could not be used, but the remaining 7 were logged and transcribed completely.

Participants completed a social dominance assessment questionnaire based on scales developed by Burgoon [7] and refined by Huang [18] for use in media assessment. The pre-experiment instrument asked participants to assess their own behavior, while the post-experiment instruments asked them to assess both of their partners' behavior, and their own. The post-experiment instrument was completed twice - once after each input condition.

\section{RESULTS}

We divide our discussion of results into three sections. The first discusses the impact of input condition on perceived social dominance and competitive behavior. The second explores differences in group process between conditions. The third summarizes general observations of how the users' seating positions might impact task performance.

\section{Social Dominance and Competitive Behavior}

Social Dominance

The performance of the social dominance scales was assessed using Cronbach's $\alpha-$ a measure of the internal correlation and reliability of the items that comprise a construct. Values $\geq .70$ are generally considered acceptable in the social sciences [29]. Two constructs ("poise" and "self-assurance") had $\alpha$ scores below this threshold and were dropped from further consideration. The remaining four constructs had $\alpha$ scores in the $.73-.86$ range.

We hypothesized that perceived social dominance would be affected by input configuration. In particular, we argued that if a single user controlled the mouse for a disproportionate amount of time in the single mouse condition, this could affect the perceived social dominance of that user. Support for this hypothesis was mixed.

There were no consistent differences in the perceived dominance of individuals from one condition to the next, as measured on the social dominance scale. However, there are a few factors worth discussing further.

The post-experiment questionnaire asked participants if one group member had emerged as a group leader. More participants reported a leader in the single-mouse condition (13) than in the multiple-mouse condition (8), but a $\chi^{2}$ analysis shows that this frequency does not depart from what would be expected due to chance, $\chi^{2}(1)=1.68, p>.1$. Nonetheless, it is worth exploring whether those nominated as leaders behaved differently than others, particularly with regard to their mouse control behavior. Four participants were nominated by other group members as leaders in the single-mouse condition (this number is smaller than 13 because the same person could be named by more than one other group member). On average, these participants were rated as higher on the "conversation control" dimension of social dominance $(M=4.63, \mathrm{SD}=.71)$ than their non-leader counterparts $(M=3.77, \mathrm{SD}=.73), \mathrm{F}(1,19)=4.44, \mathrm{p}<.05$. There were no statistically significant differences on the other dimensions of social dominance.

If only the second trial in the single-mouse condition is considered, group members nominated as leaders, on average, were responsible for more mouse activity $(M=62.25$ events, $\mathrm{SD}=39.15)$ than their non-leader counterparts $(M=26.23, \mathrm{SD}=30.05)$, which is marginally statistically significant, $\mathrm{F}(1,19)=4.19, \mathrm{p}<.1$. There was no such relationship in either multiple mouse trial.

\section{Competitive behavior}

We hypothesized that a single mouse would encourage competitive behavior, since when it was a user's turn to control the mouse (if they got a turn) they would likely act in their own interest. Interestingly enough, the results seem to point to the opposite of this hypothesis.

To analyze this empirically, we looked at all events for which an article was moved onto the front page, as this would be a good way for a user to act in their own interest. 
We calculated the number of times each article was moved onto the front page, and the proportion of times it was moved in by a user assigned to each of the three topic assignments. For example, out of the 35 times one article was moved onto the front page, it was done so by the user assigned to fruit $20 \%$ of the time, protein $35 \%$ of the time, and vegetables $45 \%$ of the time. We then looked at the percentage of the total value an article had to each of the topic assignments. So if an article was worth 4 points for fruit, 4 for protein, and 8 for vegetables, then the percentages would be $25 \%$ for fruit, $25 \%$ protein, and $50 \%$ vegetable. We then determined the correlation between these two values (Figure 3) - i.e., were articles that were more valuable to certain topics more likely to be moved to the front page by the user assigned to those topics?

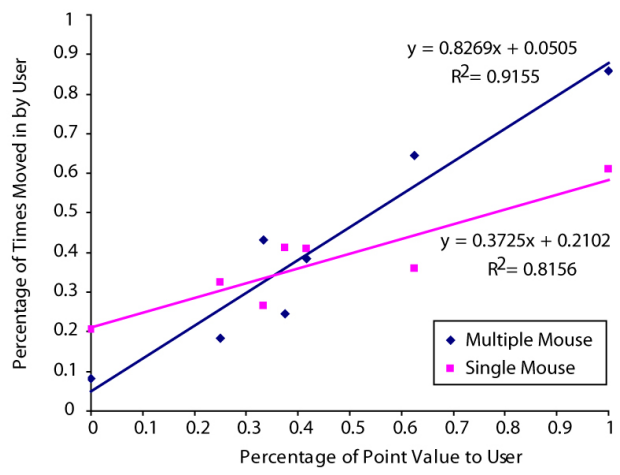

Figure 3. Scatter plot of the correlation over all groups, where dots represent articles, between the percent value of an article to a particular role (e.g., fruits, vegetables), and the fraction of the moves onto the front page for that article that were accounted for by that user.

A Student's t-test comparison of the slopes of the regression lines in the two conditions ( 0.83 multiple, 0.37 single) shows a significant difference, $\mathrm{t}(10)=3.31, \mathrm{p}<.01$. Users were acting more in their best interest in the multiple mouse condition. For example, when an article was of no value to a user, it was moved onto the front page only $8 \%$ of the time by that user in the multiple mouse condition, but $20 \%$ of the time in the single mouse condition. When an article only had value to a single user, it was moved in $86 \%$ of the time by that user in the multiple mouse condition, but only $61 \%$ of the time in the single mouse condition.

The cause for this difference in competitive behavior was seen in the video and audio transcripts. While articles dragged to the front page by users in the multi-mouse condition were rarely scrutinized by group members, they were often carefully examined - and sometimes rejected in the single mouse condition. This was particularly evident in the latter part of the experiment, where one person was typically using the mouse. Thus, the single mouse had the potential to enforce a sort of fairness via awareness of the activity of others, which is not the case with multiple mice.

Another way of analyzing competitive behavior is by looking for trials in which there were clear winners, based on the final scores. In the second trial of the single mouse condition, two participants had substantially higher (10 points or more, where the SD is 3.75) scores than the other members of their groups. Interestingly, these were not the same participants who were rated as leaders above. These "big winner" participants, however, were responsible for, on average, more mouse activity than their non-winner counterparts by a statistically significant margin $\left(M_{\text {Big Winners }}\right.$ $=94.00$ events, $\mathrm{SD}=2.83 ; M_{\text {Others }}=28.56$ events, $\mathrm{SD}=29.39), \mathrm{F}(1,34)=9.64, \mathrm{p}<.01$. There were no such relationships in the multiple mouse condition.

The "big winner" participants were also ranked higher by their group members on all social dominance dimensions, but these differences were not statistically significant, likely in part because there were only two big winners. This suggests the possibility that these individuals may have been socially dominant to begin with, and would be more likely to win under any circumstances. Interestingly, however, neither of these participants were big winners in the multiple mouse condition. Given the small number of big winners, this finding is preliminary and more research is necessary to better understand this relationship.

\section{Group Process}

Group Input

First, we wanted to know if in the multiple mouse condition participants actually made use of the devices in parallel. Video analysis showed that this was clearly the case; however we wished to quantify this parallelism. In the single mouse condition, input could never truly be parallel, but some groups may have been passing the mouse from one user to another more frequently, and we wanted to analyze this, and compare to how the mice were used in the multiple mouse condition. To do so, we need an appropriate metric to quantify parallelism. The most relevant measure in the literature is the "integrality" metric proposed by Jacob et al. [21], which took the ratio of time intervals in which events from only one input stream were recorded to time intervals in which events from multiple input streams were recorded. We extend this metric so it accounts for how many users recorded input events at each measured interval. We first split the trial into 30 second intervals. We then counted how many users had events within each of these intervals (between 0 and 3). This score would only be higher than 1 in the single mouse condition when the mouse was switched between users within a given interval. We then averaged this score across all intervals for which there was at least 1 event. The total parallelism for a trial was thus a number between 1 and 3, describing on average, how many users were using a mouse in a 30 -second interval.

Repeated measures analysis of variance showed a significant effect of input condition on parallelism $(\mathrm{F}(1,11)$ $=353, \mathrm{p}<.0001)$. The average parallelism score for multiple mice was 2.4 and for the single mouse was 1.2. This shows that users were indeed making use of the multiple mice in parallel. Figure 4 illustrates the differences in the parallel input between conditions for a typical trial. 

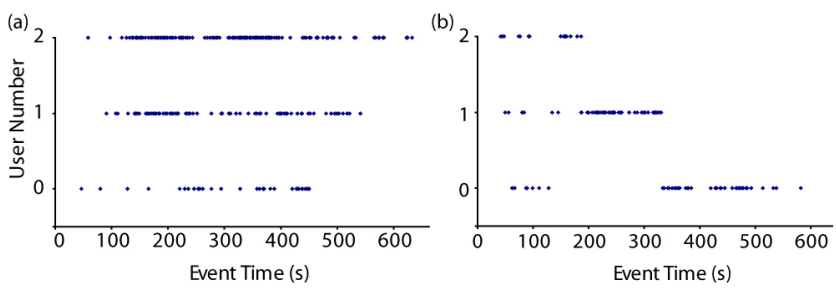

Figure 4. Scatter plots of parallel activity in a typical trial. Rows represent individual participants, whereby a dot in a row indicates user responsibility for a mouse event at the time indicated by the horizontal axis. (a): multiple mice condition. (b): single mouse condition.

To better understand what participants were doing in the single mouse condition, we looked at how often groups took turns using the mouse, versus designating (explicitly or implicitly) one user to be the controller. As Figure 5 shows, most groups used a combination of these strategies. Turn-taking happens mostly in the first quarter, while a single user tended to take over later, $(\mathrm{F}(3,18)=8.41, \mathrm{p}<$ $.05)$. Anyalsis of the videos and transcripts suggests two reasons for this. First is that groups tended to start by planning to share the mouse equally, but later implicitly allowed one person to use the mouse. The second reason stems from the nature of the task. Most groups started by individually reading and selecting the articles that were most relevant. In doing this, they passed the mouse to allow each person to select one article at a time, while the nonmouse-using users were searching for their next articles.

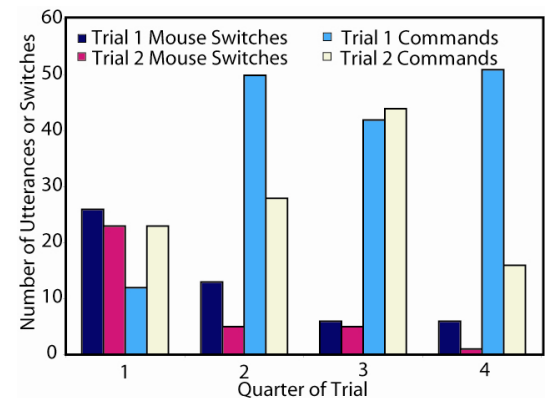

Figure 5. Number of mouse control switches between individuals (darker bars) and number of command-style utterances (lighter bars) in the four quarters of the experiment, in the Single Mouse condition only.

\section{Group Strategy}

The previous result shows that the multiple mice were used in parallel, but was this actually affecting how the task was completed? To answer this, we looked at the progression of the scores throughout the duration of a trial. Figure 6 shows the average participant score after each 30 second interval.

Repeated measures analysis of variance showed a main effect for condition on individual score $(F(1,35)=75.84$, $p$ $<.0001)$. While groups progressed through the task faster in the multiple mouse condition, the final scores do not appear to differ (Figure 6). Post hoc pair-wise mean comparisons using Tukey adjustment shows that only at interval 10 (5 minute mark) do the scores differ significantly $(\mathrm{p}=0.0039)$.
While these effects could be explained by users being able to work faster with multiple mice, we suspect the real cause is that the input condition affected the groups' overall strategy. With a single mouse, users may have elected to work together, going article to article, discussing each, and then deciding which should be moved. With multiple mice, users may individually drag articles onto the front page, and converge later as a group to decide what articles needed to be swapped in and out. This is supported by analysis of the data logs. Pair-wise comparisons show that with multiple mice scores did not change after the $10^{\text {th }}$ interval $(5 \mathrm{~min})$, while with a single mouse, scores changed significantly up to the $14^{\text {th }}$ interval $(7 \mathrm{~min})$. With multiple mice significantly more articles were both moved in $(\mathrm{F}(1,11)=9.08, \mathrm{p}<.01)$ and out $(\mathrm{F}(1,11)=7.47, \mathrm{p}<.01)$ of the front page.

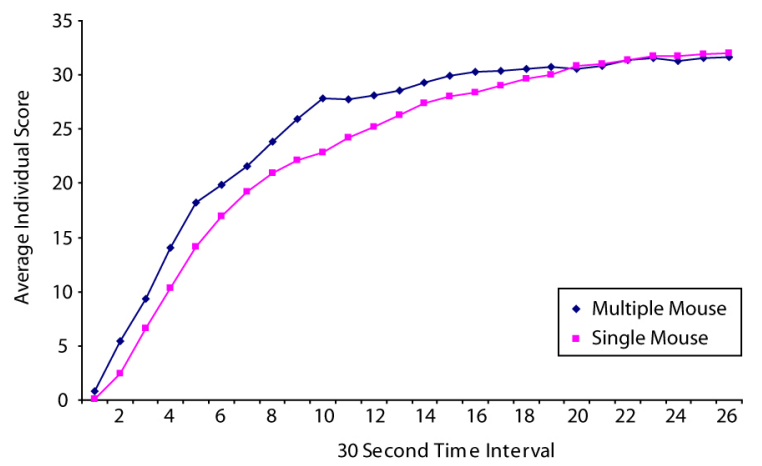

Figure 6. Progression of total (summed) individual scores over time in the two input conditions.

Group Discussion

Participants rated the quality of discussion on a seven-point Likert scale. On average discussion quality was rated higher in the single $(M=5.56, \mathrm{SD}=1.08)$ than in the multi-mouse condition $(M=4.83, \mathrm{SD}=1.61), \mathrm{F}(1,35)=6.49, \mathrm{p}<.05)$.

Transcribed utterances were counted and divided by the total time to normalize for the small number of groups that completed the task early. Overall, there were more utterances per minute in the single-mouse condition $(M=$ $12.15, \mathrm{SD}=3.47)$ than in the multiple-mouse condition $(M=$ $10.42, \mathrm{SD}=2.96), \mathrm{F}(1,6)=18.79, \mathrm{p}<.01)$. It was possible that this increase was the result of one user controlling the mouse. It was also possible that this was due to more discussion, as mouse activity could not occur in parallel. We thus analyzed the content of the conversations, coding the transcripts at least twice by two coders, with an eventual Cohen's $\kappa$ score of .75, which indicates a substantial level of agreement [8]. Categories were as follows:

Group Process: Utterances related to discussion of how to organize, proceed or manage the group's time.

Commands: Requests to the group member controlling the mouse to perform a specific action on a specific on-screen object (e.g., "make that smaller").

Task Related: Discussion of whether or not to include specific articles in the layout and their relative values. 
The number of command utterances in the single mouse condition increased sharply in the second quarter (Figure 7). Comparing this to the turn taking discussed earlier, where mouse switches decreased in the second quarter, suggests that non-mouse-controlling group members maintained their involvement in the latter three quarters.

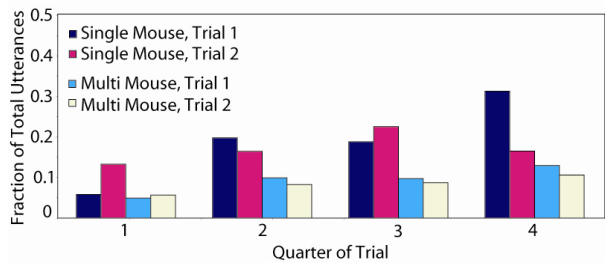

Figure 7. Fraction of command utterances in each trial of each condition, over the four quarters of the experiment.

While group process discussion occurred primarily in the first quarter, Figure 8 shows that there was generally more such discussion in the single mouse than in the multiple mouse condition, mostly in the first quarter of the first trial. This suggests that a single mouse forced groups to discuss process early. In terms of task related discussions, however, there were few, if any, substantive differences between conditions in the amount of discussion that took place.

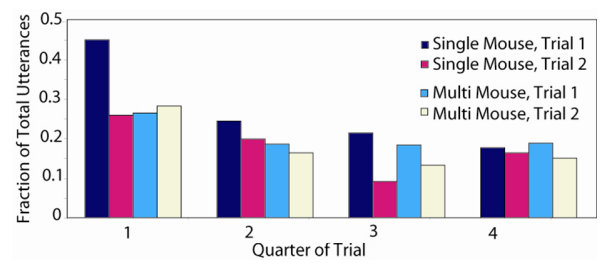

Figure 8. Fraction of group process utterances in each trial of each condition, over the four quarters of the experiment.

The transcripts also indicated frustrations caused by the single mouse, resulting in turn taking. It was easy for users to verbalize simple tasks. It was harder, however, to give more complex or vague directives, in which case it was easier to ask for control of the mouse. There were also cases where social frustration forced a change in control, as in this conflict, where $\mathrm{P} 2$ had just moved an article to the front page that benefited himself much more than the others:

P0: Wait, wait, wait...now you're pushing it

P1: That's not...

P2: That's what?

P1: That's not fair.

P0: Definitely not fair.

P2: Do something you think is fair (lets go of mouse).

P1: (grabs mouse) That I think is fair? That's fair.

\section{Seating Position and User Input Performance}

One benefit of the large display we used is that multiple users can read and interact with the same data, with roughly the same viewing clarity. However, we wondered if participants' seating position affected which portions of the screen they interacted with. Analysis of variance showed no significant affect of seating position on the $\mathrm{x}$ coordinate of articles users interacted with $(\mathrm{p}=0.57)$. Figure 9 illustrates.

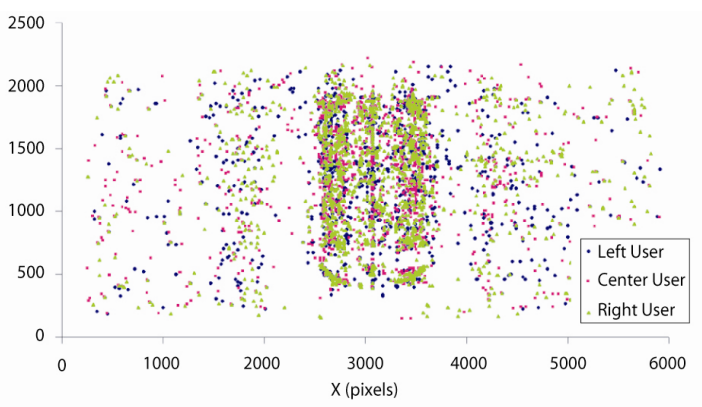

Figure 9. Scatter plot indicating mouse activity by user, by $X$ and $Y$ pixel location on the actual display.

\section{DISCUSSION}

This study has explored how input configuration might affect social dominance, competitive behavior, as well as group input, strategies, and discussions when using a large high-resolution display. Our results can provide insights for future work on multi-user large display applications.

\section{Implications for Theory}

Input condition did not affect social dominance as measured using Burgoon's [7] scale. In terms of competitive behavior and perceived leadership, however, there were differences that merit exploration. Participants who were "big winners" or rated as leaders tended in the single mouse condition (in one trial) to be responsible for more mouse events. It is, of course, not possible to reliably attribute causality from these data, but this relationship is worth exploring further.

People tended to act in their own best interest more in the multiple mouse than in the single mouse condition. This was likely because there were more command utterances in the single mouse condition, and also due to the shared focus and monitoring that a single mouse enforces.

Our analysis of parallel activity shows that single mouse control was not evenly distributed amongst users. Nonetheless, this "mouse driver" did not always (and, in fact, usually did not) dominate the negotiations, suggesting that input affects control, but control does not necessarily imply dominance. When people were paying attention and communicating with others, it was difficult for any user to dominate simply by virtue of control. Fundamentally, this comes down to van Alstyne's observation that technologies provide options, but actions create outcomes [43].

In terms of coordination, groups tended to discuss group process more in the first quarter in the single-mouse than in the multi-mouse condition. They also rated the quality of overall discussion higher in the single-mouse condition. However, they got more done faster in the multi-mouse condition. This suggests that the utility of the two input configurations may have varied by the mode of coordination required by each task component. Multiple mice supported parallel work when required, but a single mouse allowed for shared group focus when desired. While this is not entirely surprising, it suggests that future studies consider the coordination involved when selecting tasks. 
Groups adapted their coordination style to the input configuration. Where multiple mice were available, they used them for doing parallel work. With a single mouse, a team-based approach to coordination was adopted, with a single user controlling the mouse while others issued commands to that person, as it was easier than passing the mouse. This is reminiscent of the interplay between social structure and novel technologies observed by Barley [3]. This may have been different in a task with elements or operations that cannot be easily verbalized.

\section{Implications for Practice}

We observed that the mouse was an effective device for interacting with the large display. Users were able to complete the task efficiently without enhanced pointing techniques and were comfortable working with the entire display surface, regardless of their seating position..

While multiple mice allowed the groups to work in parallel, it also resulted in a lower perceived quality of discussion. The single mouse improved the quality of discussion ratings, however it sometimes caused frustration to those not controlling the mouse, and provided the opportunity for one participant to dominate the task. These results indicate that input should be considered carefully, based not only on the task but also on the group dynamics.

Key factors to consider are the structure and experience of the group. If they already have an established leader, they might respond differently to being presented with a single mouse than if there is no clear leader in that some additional negotiation may be required. Even where there is a clear leader, however, different leadership styles and cultures might also impact group response to these systems.

Similarly, groups with clearly established routines for accomplishing their tasks may also respond differently to input configuration. Our contribution here is not to suggest remedies for myriad possible scenarios, but rather to heighten awareness of the social factors at work in groups and their implications in the selection of input modalities.

\section{Limitations and Future Work}

As this is an exploratory study, there are some limitations that must be kept in mind when interpreting the results.

The experimental task has both strengths and weaknesses. Having a consistent set of objects and tasks allows for valid comparison across groups, and the task involves components of many real-world tasks, such as the need to read and understand objects in a shared visual space, and negotiating in a mixed-motive scenario. However, the task is necessarily contrived and relies on behavior that provides small financial incentives (\$5) when compared with the stakes of real world tasks. Thus, a possible limitation is that individuals did not argue for their articles as strongly as real-world users might. Nonetheless, this limitation is common in experimental economics studies [37].
Future work includes studying other input modalities, such as freehand pointing and direct pen input. We will continue to refine the tasks used in this study to better reflect realworld behavior and to more clearly differentiate between the benefits and drawbacks of different input modalities.

\section{REFERENCES}

1. Anderson, J.R. Cognitive Psychology and Its Implications. Worth Publishers, New York, NY, 2000.

2. Ball, R., Szwedo, M. and North, C. Dynamic size and speed cursor for large, high-resolution displays., Computer Science, Virginia Tech, 2006.

3. Barley, S.R. The alignment of technology and structure through roles and networks. Administrative Science Quarterly, 35, 1 (1990). 61-103.

4. Baudisch, P., Cutrell, E. and Robertson, G., highdensity cursor: a visualization technique that helps users keep track of fast-moving mouse cursors. In Proc. Interact (2003), 236-243.

5. Benko, H., Wilson, A.D. and Baudisch, P., Precise selection techniques for multi-touch screens. In Proc. CHI, (2006), 1263-1271.

6. Bezerianos, A. and Balakrishnan, R. The vacuum: facilitating the manipulation of distant objects. In Proc. CHI (2005), 361-370.

7. Burgoon, J.K., Johnson, M.L. and Koch, P.T. The nature and measurement of interpersonal dominance. Communication Monographs, 65, (1998). 308 - 335.

8. Cohen, J. A coefficient of agreement for nominal scales. Educational and Psychological Measurement, 20, 1 (1960). 37-46.

9. Czerwinski, M., Smith, G., Regan, T., Meyers, B., Robertson, G. and Starkweather, G., Woard characterizing the productivity benefits of very large displays. In Proc. Interact (2003), 9-16.

10. Davis, J. and Chen, X. Lumipoint: multi-user laserbased interaction on large tiled displays. Displays, 23, (2002). 205-211.

11. Dimicco, J.M., Pandolfo, A. and Bender, W., Influencing group participation with a shared display. In Proc. CSCW, (2004), 614-623.

12. Dudfield, H.J., Macklin, C., Fearnley, R., Simpson, A. and Hall, P., Big is better? human factors issues of large screen displays with military command teams. In People in Control (2001), 304-309.

13. Eady, P.M. and Lafferty, J.C. The subarctic survival situation. Synergistics, Plymouth, MI, 1975.

14. Elrod, S., Bruce, R., Gold, R., Goldberg, D., Halasz, F., Janssen, W., Lee, D., McCall, K., Pedersen, E., Pier, K., Tang, J.C. and Welch, B., Liveboard: a large interactive display supporting group meetings, presentations and remote collaboration. In Proc. CHI (1992), 599-607.

15. Fish, R.S., Kraut, R.E., Root, R.W. and Rice, R.E. Video as a technology for informal communication. Communications of the ACM, 36, 1 (1993). 46-61. 
16. Guimbretière, F., Stone, M. and Winograd, T., Fluid interaction with high-resolution wall-size displays. In Proc. UIST (2001), 21-30.

17. Gutwin, C. and Greenberg, S. A descriptive framework of workspace awareness for real-time groupware. Computer Supported Cooperative Work, 11, (2002). 411-446.

18. Huang, W., Olson, J.S. and Olson, G.M., Camera angle affects dominance in video-mediated communication. In Proc. CHI (2002), 716-717.

19. Inkpen, K., McGrenere, J., Booth, K.S. and Klawe, M., The effect of turn-taking protocols on children's learning in mouse-driven collaborative environments. In Proc. Graphics Interface (1997), 138-145.

20. Inkpen, K.M., Hawkey, K., Kellar, M., Mandryk, R., Parker, K., Reilly, D., Scott, S. and Whalen, T., Exploring display factors that influence co-located collaboration: angle, size, number, and user arrangement. In Proc. HCI International (2005).

21. Jacob, R.J.K., Sibert, L.E., McFarlane, D.C. and Mullen, M.P. Integrality and separability of input devices. ACM TOCHI, 1, 1 (1994). 3-26.

22. Khan, A., Fitzmaurice, G., Almeida, D., Burtnyk, N. and Kurtenbach, G., A remote control interface for large displays. In Proc. UIST (2004), 127-136.

23. Kraut, R. Applying social psychological theory to the problems of group work. in Carroll, J.M. ed. HCI Models, Theories and Frameworks, Morgan Kaufmann, New York, 2003, 325-356.

24. Malik, S., Ranjan, A. and Balakrishnan, R. Interacting with large displays from a distance with vision-tracked multi-finger gestural input. in Proc. UIST (2005), 4352.

25. Malone, T.W. and Crowston, K. The interdisciplinary study of coordination. ACM Computing Surveys, 24, 1 (1994). 87-119.

26. McGrath, J.E. Groups: Interaction and performance. Prentice-Hall, Englewood Cliffs, NJ, 1984.

27. Moran, T.P., Chiu, P., Melle, W.v. and Kurtenbach, G., Pen-based interaction techniques for organizing material on an electronic whiteboard. In Proc. UIST (1997), 127-136.

28. Morris, M.R., Ryall, K., Shen, C., Forlines, C. and Vernier, F., Beyond "social protocols:" multi-user coordination policies for co-located groupware. In Proc. CSCW (2004), 262-265.

29. Nunally, J.C. Psychometric Theory. McGraw-Hill, New York, 1978.

30. Oh, J.-Y. and Stuerzlinger, W., Laser pointers as collaborative pointing devices. In Proc. Graphics Interface (2002).

31. Olsen, D.R. and Nielsen, T., Laser pointer interaction. In Proc. CHI (2001), 17-22.

32. Olson, G.M. and Olson, J.S. Distance matters. HumanComputer Interaction, 15, (2001). 139-179.

33. Ranjan, A., Birnholtz, J.P. and Balakrishnan, R., An exploratory analysis of partner action and camera control in a video-mediated collaborative task. In Proc. CSCW (2006).

34. Renambot, L., Jeong, B., Jagodic, R., Johnson, A. and Leigh, J., Collaborative visualization using highresolution tiled displays. In ACM CHI Workshop on Information Visualization Interaction Techniques for Collaboration Across Multiple Displays (2006).

35. Robertson, G., Czerwinski, M., Baudisch, P., Meyers, B., Robbins, D., Smith, G. and Tan, D.S. The largedisplay user experience. IEEE Computer Graphics and Applications (2005). 44-51.

36. Sears, A. and Schneiderman, B. High precision touchscreens: design strategies and comparisons with a mouse. International Journal of Man-Machine Studies, 34, 4 (1991). 593-613.

37. Smith, V. Microeconomic systems as experimental science. American Economic Review, 72, 5 (1982). 923-955.

38. Stewart, J., B., B.B. and Druin, A., Single display groupware: a model for co-present collaboration. In Proc. CHI (1999), 286-293.

39. Streitz, N.A., Geissler, J., Holmer, T., Konomi, S., Muller-Tomfelde, C., Reischl, W., Rexroth, P., Seitz, P. and Steinmetz, R., i-LAND: An interactive landscape for creativity and information. In Proc. CHI (1999), 120-127.

40. Teasley, S., Covi, L.A., Krishnan, M.S. and Olson, J.S. Rapid software development through team collocation. IEEE Trans. on Software Engineering, 28, 6 (2002).

41. Thompson, J.D. Organizations in Action: Social Science Bases of Administrative Theory. McGraw-Hill, New York, 1967.

42. Tse, E. and Greenberg, S., Rapidly prototyping single display groupware through the SDG toolkit. In Proc. AUIC (2004), 101-110.

43. van Alstyne, M. and Brynjolfsson, E. Could the Internet balkanize science? Science, 274, 5292 (1996). 1479-1480.

44. Van De Ven, A.H., Delbecq, A.L. and Koenig Jr., R. Determinants of coordination modes within organizations. American Sociological Review, 41, 2 (1976). 322-338.

45. Vogel, D. and Balakrishnan, R., Distant freehand pointing and clicking on very large high resolution displays. In Proc. UIST (2005), 33-42.

46. Vogel, D. and Balakrishnan, R., Interactive public ambient displays: Transitioning from implicit to explicit, public to personal, interaction with multiple users. In Proc. UIST (2004), 137-146.

47. Vogt, F., Wong, J., Po, B., Argue, R., Fels, S.S. and Booth, K.S., Exploring collaboration with group pointer interaction. In Proc. Computer Graphics International (2004), 636-639.

48. Wilhelmson, R., Baker, P., Stein, R. and Heiland, R., Large tiled display walls and applications in meteorology, oceanography, and hydrology. In Proc. Int. Conf. on Interactive Information (2002), 29-30. 